\title{
Abductor tendon tears are associated with hypertrophy of the tensor fasciae latae muscle
}

\author{
Sutter, Reto ; Kalberer, Fabian ; Binkert, Christoph A ; Graf, Nicole ; Pfirrmann, Christian W A ; \\ Gutzeit, Andreas
}

\begin{abstract}
OBJECTIVE: To evaluate the association between hypertrophy of the tensor fasciae latae muscle and abductor tendon tears. MATERIALS AND METHODS: Thirty-five patients who underwent MRI of the abductor tendons of the hip were included in this retrospective study. A subgroup of 18 patients was examined bilaterally. The area of the tensor fasciae latae muscle and the area of the sartorius muscle (size reference) were quantified at the level of the femoral head, and a ratio was calculated. Two radiologists assessed the integrity of the gluteus medius and minimus tendon in consensus. Data were analyzed with a Mann-Whitney U test. RESULTS: Sixteen out of 35 patients (46\%) had a tear of the gluteus medius or minimus tendon. The ratio of the area of the tensor fasciae latae to the sartorius muscle was significantly higher $(\mathrm{p}=.028)$ in the group with an abductor tendon tear (median 2.25; Interquartile Range $[\mathrm{IQR}]=1.97-3.21)$ compared to the group without any tears (median $1.91 ; \mathrm{IQR}=$ 1.52-2.26). The bilateral subanalysis showed that in patients without a tear, the ratio of the two areas did not differ between each side $(\mathrm{p}=.966)$, with a median of 1.54 (primary side) and 1.76 (contralateral side). In patients with an abductor tendon tear the ratio was significantly higher $(\mathrm{p}=.031)$ on the side with a tear (median 2.81) compared to the contralateral healthy side (1.67). CONCLUSION: Patients with abductor tendon tears showed hypertrophy of the tensor fasciae latae muscle when compared to the contralateral healthy side and to patients without a tear.
\end{abstract}

DOI: https://doi.org/10.1007/s00256-012-1514-2

Posted at the Zurich Open Repository and Archive, University of Zurich

ZORA URL: https://doi.org/10.5167/uzh-85935

Journal Article

Published Version

Originally published at:

Sutter, Reto; Kalberer, Fabian; Binkert, Christoph A; Graf, Nicole; Pfirrmann, Christian W A; Gutzeit, Andreas (2013). Abductor tendon tears are associated with hypertrophy of the tensor fasciae latae muscle. Skeletal Radiology, 42(5):627-633.

DOI: https://doi.org/10.1007/s00256-012-1514-2 
Skeletal Radiology, in press

\title{
Abductor tendon tears are associated with hypertrophy of the tensor fasciae latae muscle
}

\author{
Reto Sutter ${ }^{1,2}$, Fabian Kalberer ${ }^{3}$, Christoph A. Binkert ${ }^{2,4}$, Nicole Graf ${ }^{4}$, \\ Christian W. A. Pfirmann ${ }^{1,2}$, Andreas Gutzeit ${ }^{4,5}$
}

\footnotetext{
${ }^{1}$ Department of Radiology, Orthopedic University Hospital Balgrist, Zurich, Switzerland ${ }^{2}$ University of Zurich, Zurich, Switzerland

${ }^{3}$ Department of Orthopedic Surgery, Cantonal Hospital, Winterthur, Switzerland

${ }^{4}$ Department of Radiology, Cantonal Hospital, Winterthur, Switzerland

${ }^{5}$ Department of Radiology, Paracelsus Medical University Salzburg, Salzburg, Austria
}

\section{Corresponding author:}

Reto Sutter M.D., Consultant Radiologist, Department of Radiology, University Hospital Balgrist, Forchstrasse 340, 8008 Zurich, Switzerland 


\section{Abstract}

Objective. To evaluate the association between hypertrophy of the tensor fasciae latae muscle and abductor tendon tears.

Materials and Methods. Thirty-five patients with MRI of the abductor tendons of the hip were included in this retrospective study. A subgroup of 18 patients was examined bilaterally. The area of the tensor fasciae latae muscle and the area of the sartorius muscle (size reference) were quantified at the level of the femoral head, and a ratio was calculated. Two radiologists assessed the integrity of the gluteus medius and minimus tendon in consensus. Data were analyzed with a Mann-Whitney $U$ test.

Results. Sixteen out of 35 patients $(46 \%)$ had a tear of the gluteus medius or minimus tendon. The ratio of the area of the tensor fasciae latae to the sartorius muscle was significantly higher $(p=.028)$ in the group with an abductor tendon tear (median 2.25; Interquartile Range $[\mathrm{IQR}]=1.97-3.21$ ) compared to the group without tear (median 1.91; $I Q R=1.52-2.26)$. The bilateral subanalysis showed that in patients without a tear, the ratio of the two areas did not differ between each side $(p=.966)$, with a median of 1.54 (primary side) and 1.76 (contralateral side). In patients with an abductor tendon tear the ratio was significantly higher $(p=.031)$ on the side with a tear (median 2.81) compared to the contralateral healthy side (1.67).

Conclusion. Patients with abductor tendon tears showed hypertrophy of the tensor fasciae latae muscle when compared to the contralateral healthy side and to patients without a tear.

\section{Keywords}

Abductor tendons, tensor fasciae latae, hypertrophy, hip, magnetic resonance imaging 


\section{Introduction}

The greater trochanteric pain syndrome is characterized by chronic pain and tenderness over the greater trochanter [1, 2]. Notably, this syndrome can present both in patients with and without total hip arthroplasty (THA), and it is commonly caused by abductor tendon abnormalities [1, 3-5]. In addition to severe lateral hip pain, abductor tendon tears and atrophy of the associated muscles often result in further clinical problems such as a Trendelenburg gait pattern, or immobility [6]. MRI is routinely used for evaluation of suspected abnormalities of the gluteus medius and minimus tendon and muscle, both in patients with and without THA, and with the routine use of MRI the knowledge about abductor tendon abnormalities has increased in recent years $[3,1,2,7,8]$. However, the biomechanics of the development of abductor tendon tears are still poorly understood, and the role of the tensor fasciae latae muscle in the development of abductor tendon tears has not been evaluated up to date [8].

It is known that certain muscles may undergo hypertrophy when the weight load of the affected region changes: An example for this phenomenon in the hip region is the iliocapsularis muscle, which hypertrophies in developmental hip dysplasia [9]. When reading MR examinations of the hip in daily routine, we frequently observe hypertrophy of the tensor fasciae latae muscle in patients with abductor tendon abnormalities, but to our knowledge it has not been described in the literature whether hypertrophy of the tensor fasciae latae muscle is associated with a tear of the abductor tendons.

Therefore, the purpose of this study was to evaluate the association between hypertrophy of the tensor fasciae latae muscle and abductor tendon tears. 


\section{Materials and Methods}

\section{Patients}

This retrospective study was approved by the local ethics committee. Written informed consent for retrospective use of the data was obtained from all patients as part of their hospital admission. Consecutive MRI examinations of the abductor tendons from a single center were included between July 2010 and September 2011. Both patients with and without total hip arthroplasty (THA) were included.

The following exclusion criteria were used: Patients where the tensor fasciae latae or the sartorius muscle were not completely visible on the MRI images (9 patients excluded); abductor tendon insertion not visible due to metal artifacts induced by THA ( 0 patients); age $<18$ years ( 0 patients); pregnancy ( 0 patients); no informed consent ( 0 patients).

Overall, 35 patients were included in the study. Body weight and length were recorded in each patient before the MR examination was performed, and subsequently the body mass index (BMI) was calculated (Table 1). A subgroup of 18 patients was examined bilaterally, resulting in MRI data of the symptomatic side (primary side) and an asymptomatic side (contralateral side).

\section{$\underline{\text { MR imaging }}$}

All patients were examined using a 1.5T MRI scanner (Achieva, Philips Healthcare, Best, Netherlands). Patients were positioned supine on the MR examination table and a 16 channel XL torso coil (Philips Healthcare, Best, Netherlands) was used for image acquisition. The standardized MRI protocol is summarized in Table 2.

\section{$\underline{\text { Quantitative Image Analysis }}$}

One radiologist (blinded for review purposes) selected an image at the level of the center of the femoral head for quantitative measurements on a transverse T1-weighted sequence (Fig. 1). On the selected slice he measured the cross-sectional area of the tensor fasciae 
latae muscle and of the sartorius muscle at the same level. Based on these area measurements, a ratio of the tensor fasciae latae muscle area and the ipsilateral sartorius muscle area was calculated.

Additionally, the transverse diameter of the tensor fasciae latae muscle was measured on the same image similar to the method described for the iliocapsularis muscle [10], with the transverse diameter being defined as the maximal diameter of the short axis of the muscle (Fig. 1). Then the transverse diameter of the sartorius muscle was measured with the same technique. Based on the diameter measurements, a ratio of the tensor fasciae latae muscle diameter and the sartorius muscle diameter was calculated.

\section{Qualitative Image Analysis}

Two radiologists (blinded for review purposes) assessed all examinations in a consensus reading, and were blinded towards the clinical information during the image analysis. The quality of the gluteus medius and minimus tendon was assessed on a four-point scale: 0 , normal; 1 , tendinopathy; 2 , partial tear; 3 , complete tear. Tendinopathy was defined as increased tendon diameter or hyperintensity in the T1-weighted sequence, but without intratendinous hyperintensity in the T2-weighted or fluid-sensitive sequences. A partial tear was defined as focal hyperintensity in the T2-weighted or fluid-sensitive sequences that did not involve the whole diameter of the tendon. A complete tear was defined as hyperintensity in the T2-weighted or fluid-sensitive sequences that involved the whole tendon diameter. Fatty infiltration of the gluteus medius and minimus muscle as well as the tensor fasciae latae and sartorius muscle were assessed with the Goutallier classification that has already been used in the literature for the evaluation of the abductor muscles: Grade 0, no intramuscular fat; grade 1, some fat streaks; grade 2, fatty infiltration, but less fat than muscle tissue; grade 3 , equal amounts of fat and muscle tissue; grade 4 , more fat than muscle tissue $[11,12]$. It was also noted in each patient whether THA or trochanteric bursitis was present. 


\section{Statistical Analysis}

Descriptive statistics were used to compare muscle areas and diameters, and median values and interquartile ranges (IQR) were calculated. Differences between characteristics in patients with a tear and without a tear were compared with an unpaired t-test (BMI and age differences) or a Fisher's exact test (gender, presence of THA, tear of abductor tendon, trochanteric bursitis), with $p<0.05$ indicating significance (Table 1). Differences in muscle cross-sectional area and muscle diameter were compared with a Mann-Whitney $U$ test, with $p<0.05$ indicating significance. Correlation between the tensor fasciae latae muscle area and the BMI was analyzed by using a Spearman's rank correlation coefficient. All analyses were performed with statistical software (SPSS for Windows, release 17.0; SPSS, Chicago, III). 


\section{Results}

In the group of patients with a tear (16 patients), the tears affected the gluteus minimus tendon in 10 patients $(62.5 \%)$, and the gluteus medius tendon in 14 patients $(87.5 \%)$ (Table 1). A tear of both the gluteus medius and minimus tendon was present in 8 patients $(50 \%)$, while only one of these tendons was torn in the other 8 patients $(50 \%)$. Nineteen hips showed no tear of the abductor tendons. There was no statistically significant difference between the group with a tear and without a tear regarding gender and THA (Table 1). Patients with an abductor tendon tear had a lower BMI and were older than patients without a tear, albeit with some overlap between the groups (Table 1).

\section{$\underline{\text { Cross-sectional area }}$}

In the group with an abductor tendon tear, a median cross-sectional area of the ipsilateral tensor fasciae latae muscle of $4.93 \mathrm{~cm}^{2}$ (Interquartile Range, IQR: $4.45-6.18 \mathrm{~cm}^{2}$ ) was measured at the level of the center of the femoral head. In the group without an abductor tendon tear, a median area of the ipsilateral tensor fasciae latae muscle of $5.70 \mathrm{~cm}^{2}$ (IQR: 4.31-6.99 $\mathrm{cm}^{2}$ ) was measured. There was no statistically significant difference in the area of the tensor fasciae latae muscle between the group with and without a tear $(p=0.476)$ (Fig. 2). There was a moderate correlation between the area of the tensor fasciae latae muscle and the BMI $\left(r^{2}=0.454 ; p=0.007\right)$.

\section{$\underline{\text { Ratio }}$}

The ratio of the area of the tensor fasciae latae muscle and the area of the sartorius muscle (as a size reference) was significantly higher in the group with an ipsilateral abductor tendon tear (median 2.25; IQR 1.97-3.21) compared to the group without a tear (median 1.91; IQR 1.52-2.26) $(p=0.028)($ Fig. 3, 4).

\section{Diameter}


In the group with an abductor tendon tear, a median diameter of the tensor fasciae latae muscle of $1.73 \mathrm{~cm}$ was measured at the level of the center of the femoral head. In the group without an abductor tendon tear, a median diameter of the tensor fasciae latae muscle of $1.70 \mathrm{~cm}$ was measured. There was no difference in the diameter of the tensor fasciae latae muscle between the group with and without a tear $(p=0.800)$. There was no significant difference between the ratio of the diameter of the tensor fasciae latae and the sartorius muscle between the group with and without an abductor tendon tear $(p=0.260)$.

\section{Bilateral subanalysis}

In the group with an abductor tendon tear, the cross-sectional area of the ipsilateral tensor fasciae latae was $5.50 \mathrm{~cm}^{2}$ (IQR: $\left.4.10-6.18 \mathrm{~cm}^{2}\right)$, while on the contralateral side the area was only $3.80 \mathrm{~cm}^{2}$ (IQR: $\left.2.80-4.94 \mathrm{~cm}^{2}\right)$. However, this was not statistically significant $(p=$ 0.096). In the group without an abductor tendon tear, the area of the ipsilateral tensor fasciae latae was $5.30 \mathrm{~cm}^{2}$ (IQR: $4.30-5.70 \mathrm{~cm}^{2}$ ), and for the contralateral side the area was $5.00 \mathrm{~cm}^{2}$ (IQR: $\left.4.35-5.25 \mathrm{~cm}^{2}\right)$, with $p=0.818$.

In the group with an abductor tendon tear, the cross-sectional area of the ipsilateral sartorius was $1.95 \mathrm{~cm}^{2}$ (IQR: $1.72-2.35 \mathrm{~cm}^{2}$ ), and on the contralateral side the area was $2.09 \mathrm{~cm}^{2}$ (IQR: $1.90-2.40 \mathrm{~cm}^{2}$ ), with $p=0.370$. In the group without an abductor tendon tear, the area of the ipsilateral sartorius was $3.30 \mathrm{~cm}^{2}\left(\right.$ IQR: $\left.2.72-3.75 \mathrm{~cm}^{2}\right)$, and on the contralateral side the area was $3.20 \mathrm{~cm}^{2}\left(\mathrm{IQR}: 2.50-3.45 \mathrm{~cm}^{2}\right)$, with $p=0.844$.

The bilateral subanalysis showed that in patients without a tear $(n=11)$, the ratio of the two areas did not differ between the symptomatic side (primary side) and the asymptomatic healthy side (contralateral side) $(p=0.966)$, with a median of 1.54 on the primary side (IQR: $1.46-1.80)$ and a median of 1.76 on the contralateral side (IQR: 1.47-1.81). However, in patients where an abductor tendon tear was present $(n=7)$ the ratio was significantly higher on the side with a tear (median 2.81; IQR 1.97-3.22) compared to the contralateral healthy side (median 1.67; IQR 1.53-1.90) $(p=0.031)$ (Fig. 5). 


\section{Fatty infiltration}

In patients with an abductor tendon tear, there was significantly increased fatty infiltration of the ipsilateral gluteus medius and minimus muscle $(p=0.007$ and $p=0.002$, respectively), compared to patients without a tear (Table 3). Conversely, in patients with an abductor tendon tear there was slightly less fatty infiltration of the ipsilateral tensor fasciae latae muscle, compared to patients without a tear, but this was not statistically significant $(p=0.182)$ (Table 3). 


\section{Discussion}

We were able to show that the ipsilateral tensor fasciae latae muscle is hypertrophied in patients with tears of the gluteus medius or gluteus minimus tendon. This finding suggests that the tensor fasciae latae muscle adopts a compensatory function in patients with an impaired hip abduction due to a gluteal tendon tear. Due to a substantial variability in the size of the tensor fasciae muscle both in our data and in measurements in cadavers [13], and due to a moderate correlation of the cross-sectional area of the tensor fasciae latae muscle with the BMI, an isolated comparison of the area in patients with and without a tear did not prove helpful in assessing a possible hypertrophy of the muscle. When the cross-sectional area of the ipsilateral sartorius muscle was introduced as a size reference and a ratio was calculated between the area of the tensor fasciae latae muscle and the sartorius muscle, this ratio was statistically significantly larger in patients with a tear of the abductor tendons (median 2.25) than in patients without a tear (median 1.91). These findings were confirmed in the subanalysis of patients with bilateral MRI: Here the ratio showed a median of 2.81 on the side with an abductor tendon tear and of 1.67 on the contralateral healthy side.

While the ratio of the areas of the two muscles was larger in patients with an abductor tendon tear compared to the patients without a tear, there was a substantial overlap between the two groups. Therefore, a single cut-off value for the ratio is not able to distinguish correctly between patients with and without abductor tendon tears. Our data further show that neither the short-axis diameter of the tensor fasciae latae nor the ratio between the short-axis of the tensor fasciae latae and the sartorius muscle were able to reliably indicate hypertrophy of the tensor fasciae latae muscle.

The tensor fasciae latae muscle is part of the hip abductor muscle group and is involved in the stabilization of the pelvis during gait, although clinically the term 'hip abductors' is mostly utilized as a collective term for the gluteus medius and minimus muscle $[14,15]$. The tensor fasciae latae muscle originates at the anterior lateral portion of the iliac crest 
and joins the middle layer of the fascia lata distally [16]. While the tensor fasciae latae muscle is less commonly injured than the other hip abductors [17], a recent report using electromyographic recordings showed that patients with symptomatic femoroacetabular impingement had a reduced ability to activate the tensor fasciae latae muscle during hip flexion [18], showing that the tensor fasciae latae can undergo changes due to a different weight loading.

There was slightly less fatty infiltration of the tensor fasciae latae muscle in patients with abductor tendon tears than in patients without tears in our study. This can be explained by the way muscle tissue reacts to a change in weight load: A muscle or portions of a muscle can undergo hypertrophy with increased weight loading [19, 9]. Conversely, decreased weight loading is associated with muscle hypotrophy and fatty infiltration [20, 11, 21]. It is known that a different amount of traumatic injury occurs depending on the surgical approach that is chosen in patients with THA $[10,22,23,7]$. A recent report showed compensatory hypertrophy of the tensor fasciae latae muscle in patients with the lateral approach, when compared to the anterolateral approach in THA patients, due to increased muscle trauma to the gluteus medius [24]. This strengthens our findings, even though the gluteal tendon integrity was not assessed in that study [24].

Our study underlines the importance of the tensor fasciae latae muscle in patients with tears of the gluteus medius and minimus muscle. As a possible clinical consequence, orthopedic surgeons might try to preserve the tensor fasciae latae muscle from damage by selecting a surgical access that avoids injuring this muscle in patients where a reconstruction of a torn gluteus tendon is performed. Moreover, our results suggest that if hypertrophy of the tensor fasciae latae muscle is detected at computed tomography or sonography in patients with lateral hip pain, a tear of the gluteus medius or minimus tendon should be suspected and the abductor tendons might be evaluated with a subsequent MR examination. 
This study had limitations. The MRI data were analyzed retrospectively, and there was no surgical confirmation for the tears of the abductor tendons that were seen on MRI. The number of subjects included in the study is relatively small. Further, our control group did not consist of asymptomatic age-matched healthy volunteers, which would have been a better reference standard.

In conclusion, patients with abductor tendon tears showed hypertrophy of the tensor fasciae latae muscle when compared to the contralateral healthy side and to patients without a tear. 
Figures

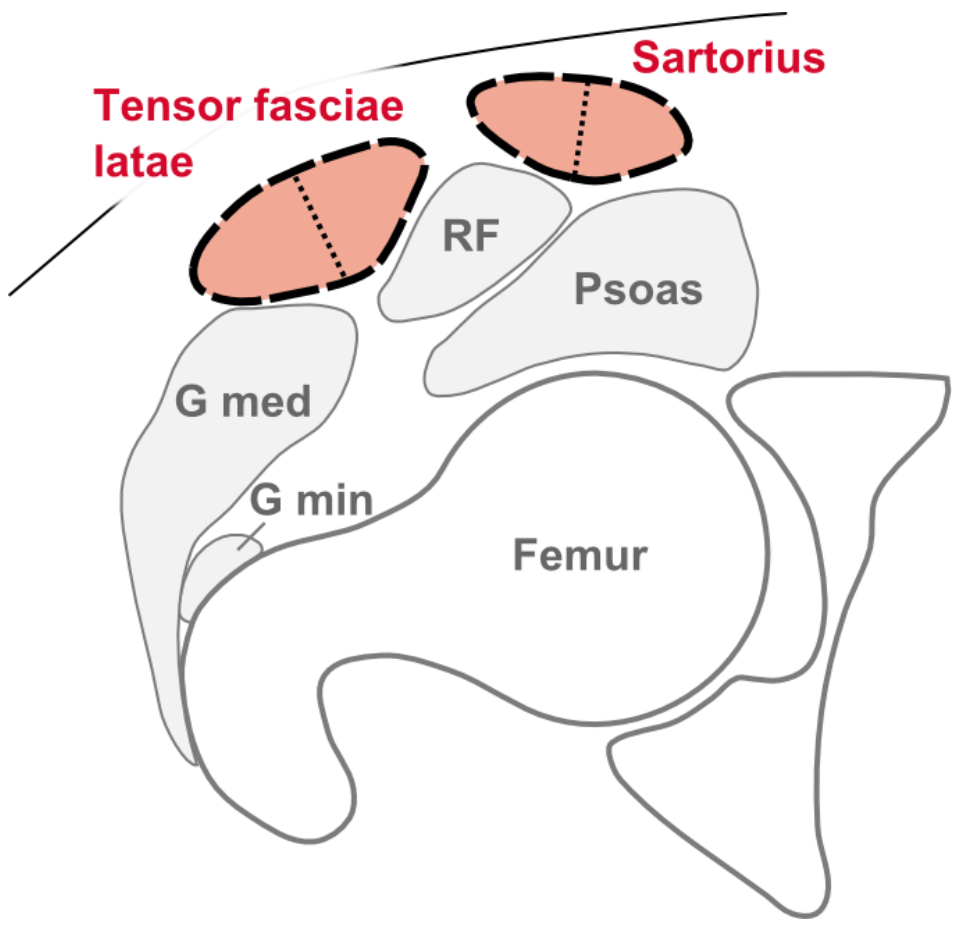

Fig. 1 Schematic figure of area and diameter measurements of the tensor fasciae latae muscle and the sartorius muscle on a transverse image of the right hip. Dashed lines outline the area of the tensor fasciae latae and the sartorius muscle at the level of the center of the femoral head. Further, the short axis diameter of each muscle is measured. For better orientation the outline of the proximal rectus femoris (RF) and of the psoas muscle is depicted, as well as the distal portion of the gluteus minimus ( $G$ min) and gluteus medius muscle (G med). 


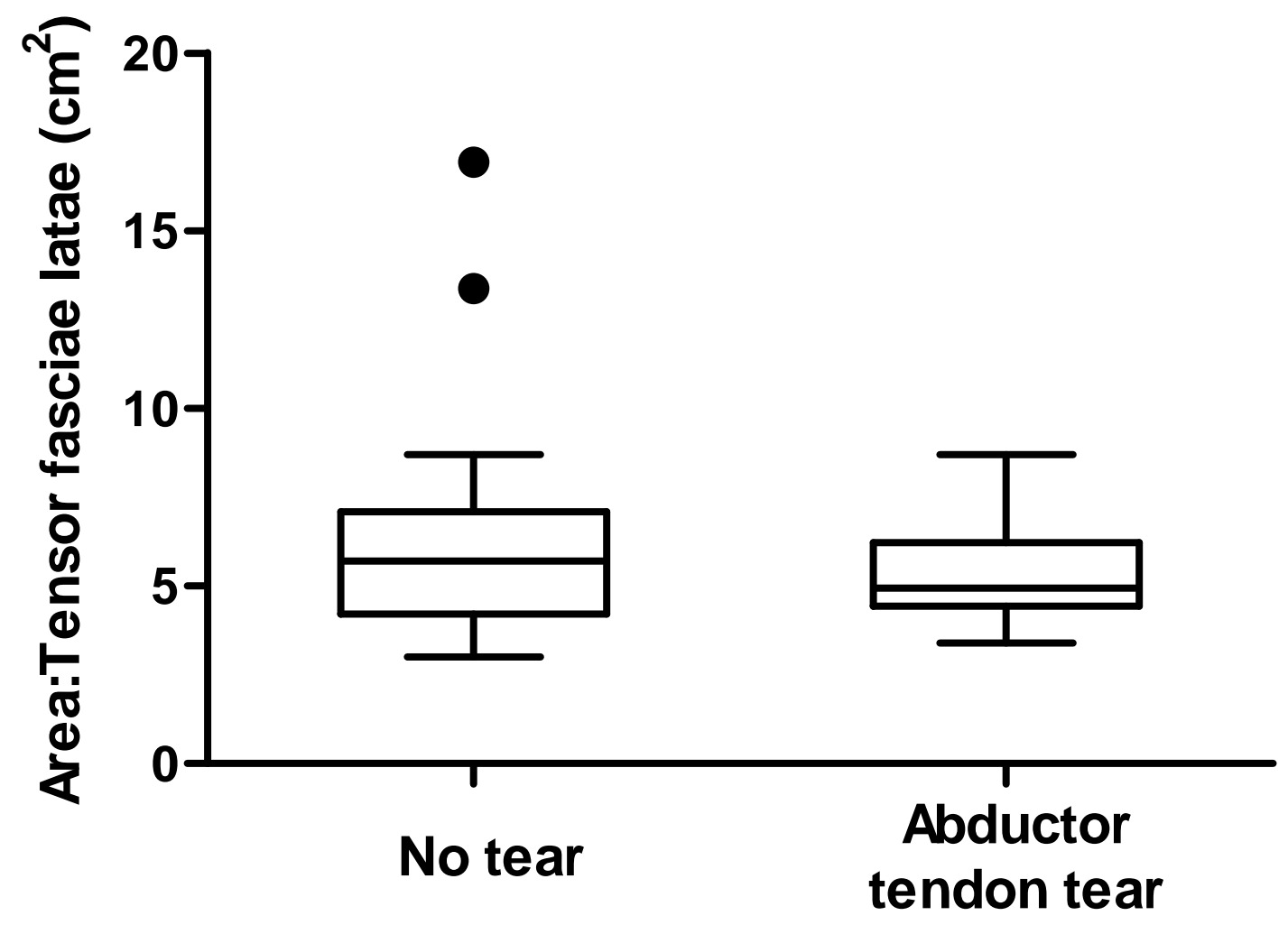

Fig. 2 The cross-sectional area of the tensor fasciae latae muscle was similar in patients without and with an abductor tendon tear $(p=0.476)$.

Note - Boxplots show the median as a thick horizontal line, and solid boxes include the first to third quartile. Whiskers extend to the farthest points that are not outliers (i.e. points within $3 / 2$ times the interquartile range), and dots indicate outliers 


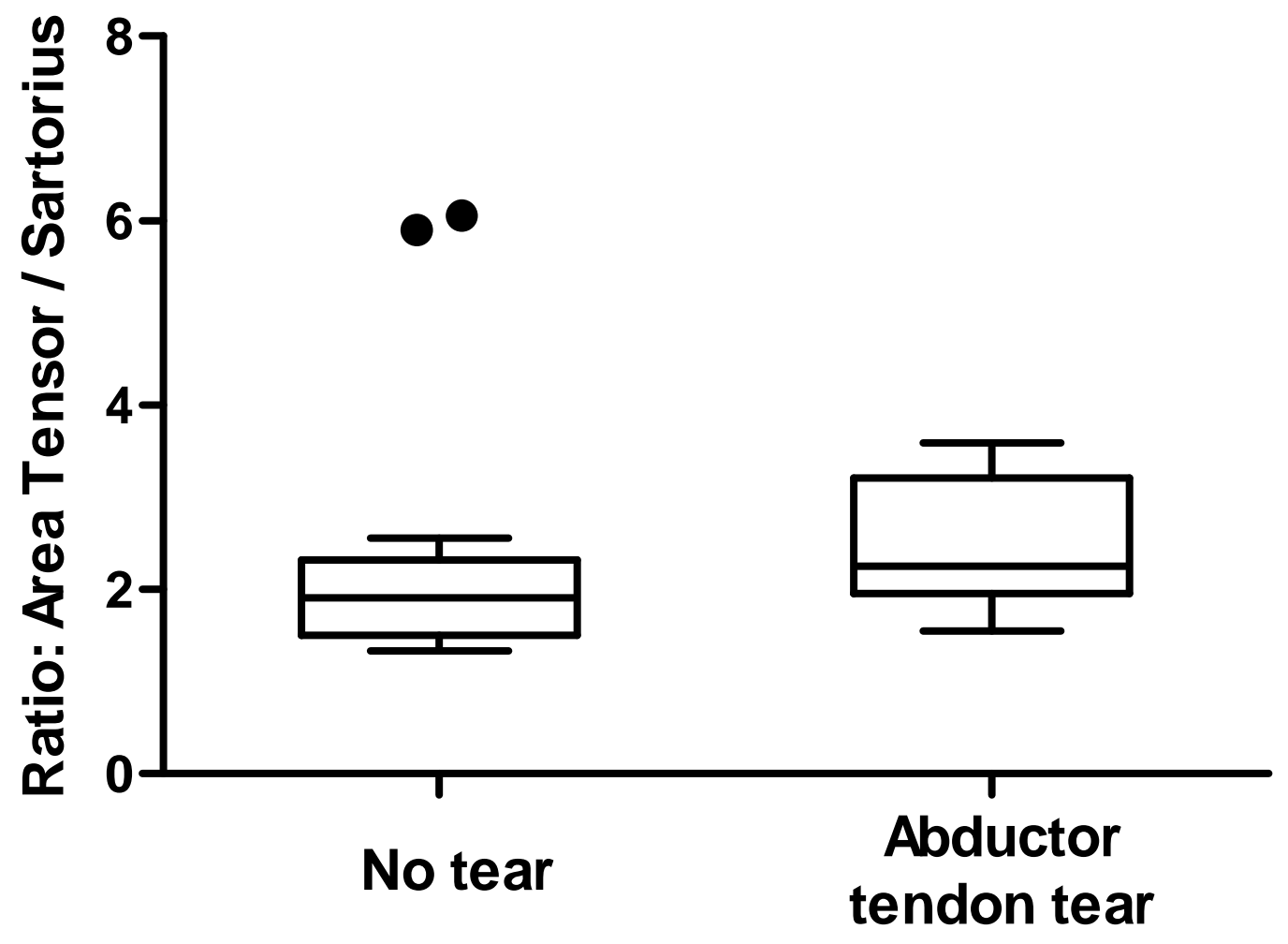

Fig. 3 The ratio of the area of the tensor fasciae latae muscle and the area of the sartorius muscle was significantly increased in the group with an abductor tendon tear compared to the group without a tear $(p=0.028)$.

Note - Boxplots show the median as a thick horizontal line, and solid boxes include first to third quartile. Whiskers extend to the farthest points that are not outliers (i.e. points within $3 / 2$ times the interquartile range), and dots indicate outliers. 

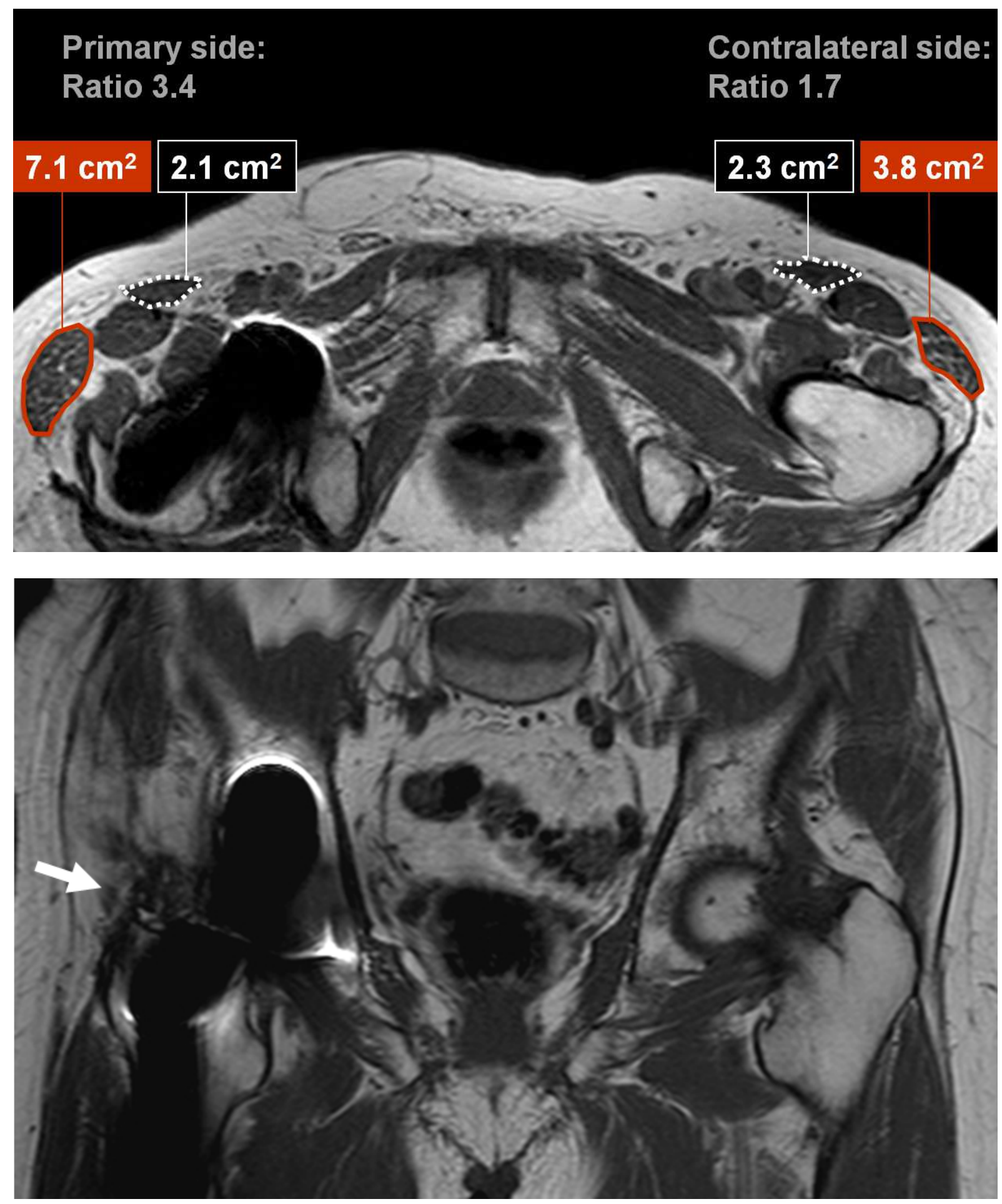

Fig. 4 Hypertrophy of the right tensor fasciae latae muscle in a 77-year-old male patient with right-sided lateral hip pain due an abductor tendon tear. (a) Bilateral area measurements for the tensor fasciae latae muscle (red outline) and the sartorius muscle (dashed white outline) on the primary (symptomatic) side and the contralateral healthy side. Transverse T1-weighted image at the level of the center of the femoral head. The 
ratio of the area of the tensor fasciae latae muscle and the sartorius muscle is 3.4 on the primary side and 1.7 on the contralateral healthy side. (b) Avulsion of the gluteus medius tendon from the lateral facet of the greater trochanter (arrow) on the right side. Coronal T2-weighted image at the level of the greater trochanter. 


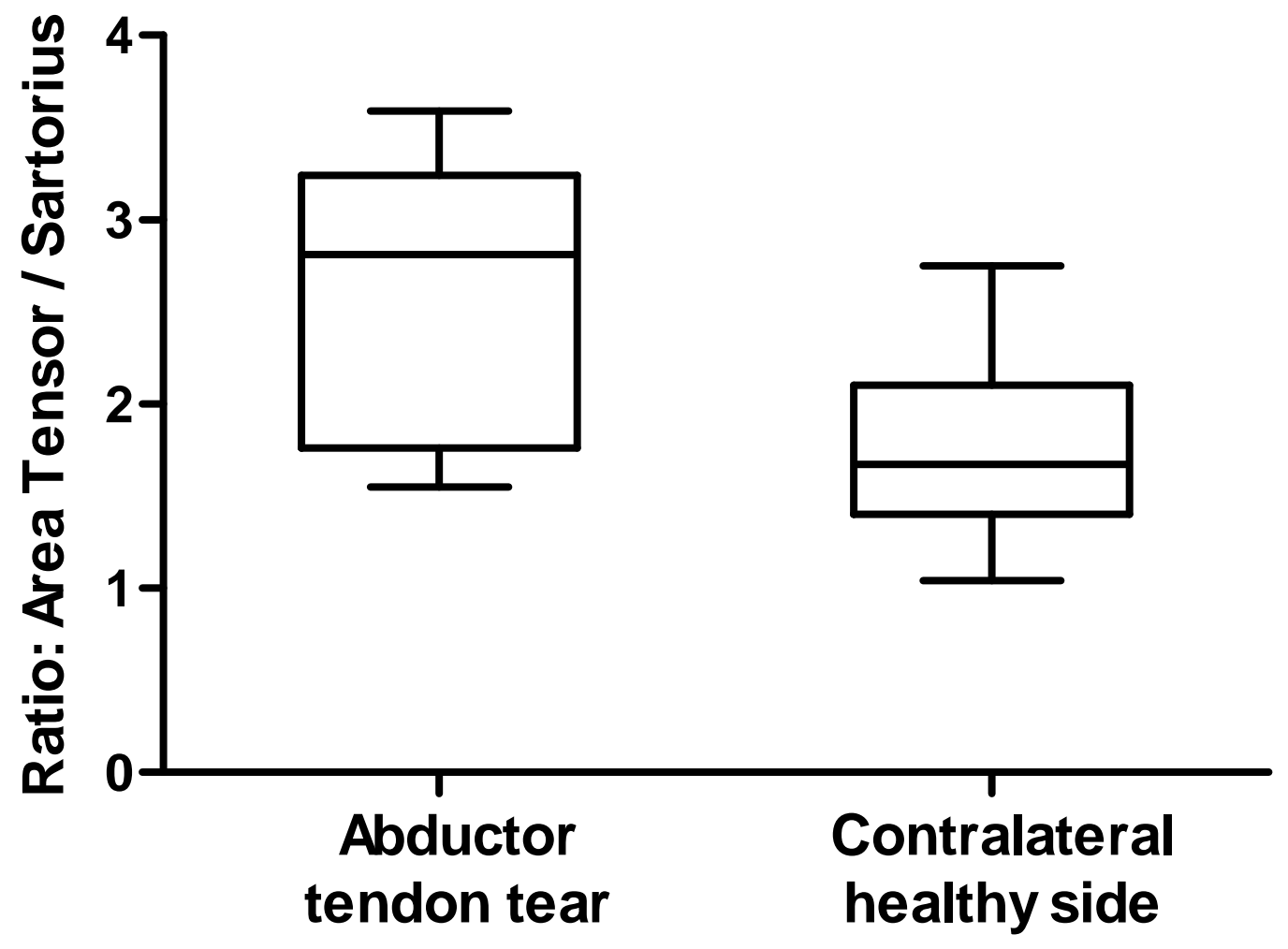

Fig. 5 In the bilateral subanalysis the ratio of the area of the tensor fasciae latae muscle and the area of the sartorius muscle was significantly increased on the side with a tear compared to the contralateral healthy side $(p=0.031)$.

Note - Boxplots show the median as a thick horizontal line, and solid boxes include first to third quartile. Whiskers extend to the farthest points that are not outliers (i.e. points within $3 / 2$ times the interquartile range), and dots indicate outliers. 
Table 1: Overview of basic characteristics according to presence of an abductor tendon tear

\begin{tabular}{|c|c|c|c|}
\hline Characteristic & $\begin{array}{l}\text { Patients with tear } \\
\qquad(n=16)\end{array}$ & $\begin{array}{l}\text { Patients without tear } \\
\qquad(n=19)\end{array}$ & significance \\
\hline Female patients & $13(81.3 \%)$ & $11(57.9 \%)$ & $p=0.2$ \\
\hline Patients with THA & $9(56.3 \%)$ & $9(47.4 \%)$ & $p=0.7$ \\
\hline $\begin{array}{l}\mathrm{BMI}\left[\mathrm{kg} / \mathrm{cm}^{2}\right] \\
\text { mean } \pm \mathrm{SD}\end{array}$ & $23.9 \pm 2.1$ & $27.5 \pm 4.9$ & $p=0.01^{a}$ \\
\hline $\begin{array}{l}\text { Age [years] } \\
\text { mean } \pm S D\end{array}$ & $71.8 \pm 10.7$ & $58.5 \pm 18.2$ & $p=0.02^{a}$ \\
\hline $\begin{array}{l}\text { Tear of gluteus } \\
\text { minimus tendon }\end{array}$ & $10(62.5 \%)$ & - & - \\
\hline $\begin{array}{l}\text { Tear of gluteus } \\
\text { medius tendon }\end{array}$ & $14(87.5 \%)$ & - & - \\
\hline Trochanteric bursitis & $10(62.5 \%)$ & $5(26.3 \%)$ & $p=0.04^{a}$ \\
\hline
\end{tabular}


Table 2: Sequence parameters for the MRI examinations

\begin{tabular}{|l|c|c|c|c|}
\hline Parameter & Transverse T1 & Sagittal T1 & $\begin{array}{c}\text { Transverse } \\
\text { STIR }\end{array}$ & Coronal T2 \\
\hline TR [ms] & 621 & 750 & 3042 & 3042 \\
\hline TE $[\mathrm{ms}]$ & 7 & 7 & 50 & 50 \\
\hline FOV [mm] & $240 \times 349 \times 447$ & $180 \times 452 \times 66$ & $170 \times 170 \times 201$ & $170 \times 170 \times 201$ \\
\hline $\begin{array}{l}\text { Acquisition time } \\
\text { [min:sec] }\end{array}$ & $4: 07$ & $6: 16$ & $3: 30$ & $3: 30$ \\
\hline
\end{tabular}

Note - TR, repetition time; TE, echo time; FOV, field of view; STIR, short tau inversion recovery. 
Table 3: Relationship between abductor tendon tears and fatty infiltration of muscles

\begin{tabular}{|c|c|c|}
\hline \multirow{2}{*}{$\begin{array}{l}\text { Fatty infiltration } \\
\text { Tensor fasciae latae }\end{array}$} & \multicolumn{2}{|c|}{ Abductor tendons } \\
\hline & No tear & Tear \\
\hline 0 & 0 & 0 \\
\hline 1 & $6(31.6 \%)$ & 9 (56.3\%) \\
\hline 2 & $13(68.4 \%)$ & $7(43.8 \%)$ \\
\hline 3 & 0 & 0 \\
\hline 4 & 0 & 0 \\
\hline Gluteus medius & No tear & Tear \\
\hline 0 & $2(10.5 \%)$ & 0 \\
\hline 1 & $10(52.6 \%)$ & $4(25.0 \%)$ \\
\hline 2 & $7(36.8 \%)$ & $8(50.0 \%)$ \\
\hline 3 & 0 & $1(6.3 \%)$ \\
\hline 4 & 0 & $3(18.8 \%)$ \\
\hline Gluteus minimus & No tear & Tear \\
\hline 0 & $2(10.5 \%)$ & 0 \\
\hline 1 & $6(31.6 \%)$ & 0 \\
\hline 2 & $4(21.1 \%)$ & $3(18.8 \%)$ \\
\hline 3 & $4(21.1 \%)$ & $5(31.3 \%)$ \\
\hline 4 & $3(15.8 \%)$ & $8(50.0 \%)$ \\
\hline
\end{tabular}

Note - Fatty infiltration of muscles was rated according to the Goutallier classification:

Grade 0 , no intramuscular fat; grade 1, some fat streaks; grade 2, fatty infiltration, but less fat than muscle tissue; grade 3, equal amounts of fat and muscle tissue; grade 4, more fat than muscle tissue. 


\section{References}

1. Cvitanic O, Henzie G, Skezas N, Lyons J, Minter J. MRI diagnosis of tears of the hip abductor tendons (gluteus medius and gluteus minimus). AJR Am J Roentgenol. $2004 ; 182(1): 137-43$.

2. Dwek J, Pfirrmann C, Stanley A, Pathria M, Chung CB. MR imaging of the hip abductors: normal anatomy and commonly encountered pathology at the greater trochanter. Magn Reson Imaging Clin N Am. 2005;13(4):691-704, vii.

3. Kingzett-Taylor A, Tirman PF, Feller J, McGann W, Prieto V, Wischer T et al. Tendinosis and tears of gluteus medius and minimus muscles as a cause of hip pain: MR imaging findings. AJR Am J Roentgenol. 1999;173(4):1123-6.

4. Miozzari HH, Dora C, Clark JM, Notzli HP. Late repair of abductor avulsion after the transgluteal approach for hip arthroplasty. J Arthroplasty. 2010;25(3):450-7 e1 .

5. Wylde V, Hewlett S, Learmonth ID, Dieppe P. Persistent pain after joint replacement: prevalence, sensory qualities, and postoperative determinants. Pain. 2011;152(3):566-72.

6. Twair A, Ryan M, O'Connell M, Powell T, O'Byrne J, Eustace S. MRI of failed total hip replacement caused by abductor muscle avulsion. AJR Am J Roentgenol. 2003;181(6):1547-50.

7. Bremer AK, Kalberer F, Pfirrmann CW, Dora C. Soft-tissue changes in hip abductor muscles and tendons after total hip replacement: comparison between the direct anterior and the transgluteal approaches. J Bone Joint Surg Br. 2011;93(7):886-9.

8. Lachiewicz PF. Abductor tendon tears of the hip: evaluation and management. J Am Acad Orthop Surg. 2011;19(7):385-91. 
9. Babst D, Steppacher SD, Ganz R, Siebenrock KA, Tannast M. The iliocapsularis muscle: an important stabilizer in the dysplastic hip. Clin Orthop Relat Res. $2011 ; 469(6): 1728-34$.

10. Walde TA, Blattgerste D, Sehmisch S, Kuttler W, Walde HJ, Koster G. Early results and patient satisfaction after total hip arthroplasty using a minimally invasive anterolateral approach. Hip Int. 2009;19(4):367-71.

11. Goutallier D, Postel JM, Bernageau J, Lavau L, Voisin MC. Fatty infiltration of disrupted rotator cuff muscles. Rev Rhum Engl Ed. 1995;62(6):415-22.

12. Pfirrmann CW, Notzli HP, Dora C, Hodler J, Zanetti M. Abductor tendons and muscles assessed at MR imaging after total hip arthroplasty in asymptomatic and symptomatic patients. Radiology. 2005;235(3):969-76.

13. Friederich JA, Brand RA. Muscle fiber architecture in the human lower limb. J Biomech. 1990;23(1):91-5.

14. Kumagai M, Shiba N, Higuchi F, Nishimura H, Inoue A. Functional evaluation of hip abductor muscles with use of magnetic resonance imaging. J Orthop Res. $1997 ; 15(6): 888-93$

15. Anderson FC, Pandy MG. Individual muscle contributions to support in normal walking. Gait Posture. 2003;17(2):159-69.

16. Pare EB, Stern JT, Jr., Schwartz JM. Functional differentiation within the tensor fasciae latae. A telemetered electromyographic analysis of its locomotor roles. J Bone Joint Surg Am. 1981;63(9):1457-71.

17. Flack NA, Nicholson HD, Woodley SJ. A review of the anatomy of the hip abductor muscles, gluteus medius, gluteus minimus, and tensor fascia lata. Clin Anat. 2012 online before print. 
18. Casartelli NC, Maffiuletti NA, Item-Glatthorn JF, Staehli S, Bizzini M, Impellizzeri FM et al. Hip muscle weakness in patients with symptomatic femoroacetabular impingement. Osteoarthritis Cartilage. 2011;19(7):816-21.

19. Soares JM. Effects of training on muscle capillary pattern: intermittent vs continuous exercise. J Sports Med Phys Fitness. 1992;32(2):123-7.

20. Williams MD, Ladermann A, Melis B, Barthelemy R, Walch G. Fatty infiltration of the supraspinatus: a reliability study. J Shoulder Elbow Surg. 2009;18(4):581-7.

21. Hoffmann A, Mamisch N, Buck FM, Espinosa N, Pfirrmann CW, Zanetti M. Oedema and fatty degeneration of the soleus and gastrocnemius muscles on MR images in patients with Achilles tendon abnormalities. Eur Radiol. 2011;21(9):1996-2003.

22. Laffosse JM, Chiron P, Molinier F, Bensafi H, Puget J. Prospective and comparative study of the anterolateral mini-invasive approach versus minimally invasive posterior approach for primary total hip replacement. Early results. Int Orthop. 2007;31(5):597-603.

23. Sendtner E, Borowiak K, Schuster T, Woerner M, Grifka J, Renkawitz T. Tackling the learning curve: comparison between the anterior, minimally invasive (Micro-hip(R)) and the lateral, transgluteal (Bauer) approach for primary total hip replacement. Arch Orthop Trauma Surg. 2011;131(5):597-602.

24. Muller M, Tohtz S, Dewey M, Springer I, Perka C. Evidence of reduced muscle trauma through a minimally invasive anterolateral approach by means of MRI. Clin Orthop Relat Res. 2010;468(12):3192-200. 